\title{
First-passage times of regime switching models ${ }^{1}$
}

\author{
Peter Hieber \\ Lehrstuhl für Finanzmathematik (M13), \\ Technische Universität München, \\ Parkring 11, 85748 Garching-Hochbrück, Germany, \\ phone $(+0049)$ 89-289-17414, fax $(+0049)$ 89-289-17407, \\ email: hieber@tum.de,
}

\begin{abstract}
The probability of a stochastic process to first breach an upper and/or a lower level is an important quantity for optimal control and risk management. We present those probabilities for regime switching Brownian motion. In the 2- and 3-state model, the Laplace transform of the (single and double barrier) first-passage times is - up to the roots of a polynomial of degree 4 (respectively 6) - derived in closed-form by solving the matrix Wiener-Hopf factorization ${ }^{2}$. This extends single barrier results in the 2-state model by Guo [2001b]. If the quotient of drift and variance is constant over all states, we show that the Laplace transform can even be inverted analytically.

Keywords: regime switching, Markov switching, first-passage time, first-exit time, (matrix) Wiener-Hopf factorization, option pricing.

Classification: 60G40, 60J27, 60J60.
\end{abstract}

After Hamilton [1989]'s seminal work, the natural and intuitive idea of regime changes has found applications in a variety of fields, ranging from biology, physics, finance, and insurance to disciplines like hydrology. Conceptionally, regime switching models are rather simple (conditional on the regimes, the innovations are normally distributed) and thus analytically tractable. Nevertheless, they can generate many non-linear effects like heavy tails or volatility clusters. Regime switching models allow us to depart from the unsatisfactory assumption of stationary increments in Lévy models and are thus a tractable tool to include long-term trends and structural breaks.

This paper derives analytical expressions for the single and double barrier first-passage time probabilities of regime switching models. Therefore, the matrix Wiener-Hopf factorization introduced by, for example, London et al. [1982], Kennedy and Williams [1990], Barlow et al.

${ }^{1}$ This version may differ from the final published version First-passage times of regime switching models, Statistics 83 Probability Letters, Vol. 92 (2014), pp. 148-157, in typographical detail.

${ }^{2}$ The matrix Wiener-Hopf factors of regime switching models are defined via a set of quadratic matrix equations (see, e.g., London et al. [1982], Barlow et al. [1990], Kennedy and Williams [1990], Rogers [1994], Asmussen [1995]). This concept was expanded to regime switching jump diffusions by Jiang and Pistorius [2008]. 


\section{Model description}

[1990], Rogers [1994], Asmussen [1995], and Jiang and Pistorius [2008] is solved analytically for the 2- and 3-state model. This yields closed-form results for the Laplace transform of the first-passage times. Imposing a parameter restriction (the quotient of drift and variance is constant over all states), we are even able to invert this Laplace transform analytically. This contributes to the analytical tractability of regime switching models and might help to increase the popularity of this model class. Related to this work is Guo [2001b] who derived the Laplace transform of the single barrier first-passage time in the 2-state model, and Jiang and Pistorius [2008] who present the single and double barrier first-passage time probabilities in terms of the matrix Wiener-Hopf factorization.

Many authors recently worked on numerical techniques to derive the first-passage time probabilities of regime switching models. Boyle and Draviam [2007], Kim et al. [2008] (and many others) solve the first-passage time PDE numerically. Hieber and Scherer [2010] and Henriksen [2011] use a conditional Monte-Carlo technique called Brownian bridge algorithm. Furthermore, some authors work on (matrix) Wiener-Hopf factorizations (see, e.g., London et al. [1982], Barlow et al. [1990], Boyarchenko and Levendorskii [2008], Jiang and Pistorius [2008], Kudryavtsev and Levendorskii [2012], Mijatović and Pistorius [2013], Fourati [2012], and others) and solve them numerically (see, e.g., Rogers and Shi [1994], Boyarchenko and Levendorskii [2008], Kudryavtsev [2010]). Kou and Wang [2003] point out that "in general, explicit calculation of the Wiener-Hopf factorization is difficult". Its derivation has turned out to be possible in the related case of exponential jump-diffusion models, i.e. the Cramér-Lundberg model, single, double, and hyper-exponential jump-diffusion models (see, e.g., Mordecki [1999], Rogers [2000], Kou and Wang [2003], Avram et al. [2003], Cai [2009]). Up to a time change, those models can be seen as special cases of regime switching models: Positive or negative exponential jumps are included as an additional state with zero volatility and positive, respectively negative, drift (a technique called "fluid embedding", see, e.g., Jiang and Pistorius [2008]).

The paper is organized as follows: Section 1 introduces regime switching models; Section 2 the matrix Wiener-Hopf factorization. The main theoretical results on the first-passage times of regime switching models are given in Section 3. Section 4 presents a numerical example. Finally, Section 5 concludes.

\section{Model description}

On the filtered probability space $(\Omega, \mathbb{F}, \mathcal{F}, \mathbb{P})$, we consider the process $B=\left\{B_{t}\right\}_{t \geq 0}$ described by the stochastic differential equation (sde)

$$
d B_{t}=\mu_{Z_{t}} d t+\sigma_{Z_{t}} d W_{t}, \quad B_{0}=x
$$

where $Z=\left\{Z_{t}\right\}_{t \geq 0} \in\{1,2, \ldots, M\}$ is a time-homogeneous Markov chain with intensity matrix $^{3} Q_{0}$ and $W=\left\{W_{t}\right\}_{t \geq 0}$ an independent Brownian motion. The initial value is $B_{0}=x \in$ $\mathbb{R}$. The filtration $\mathbb{F}=\left\{\mathcal{F}_{t}\right\}_{t \geq 0}$ is generated by the pair $(W, Z)$, i.e. $\mathcal{F}_{t}=\sigma\left\{W_{s}, Z_{s}: 0 \leq s \leq t\right\}$. The time to a state change from the current state $i$ is an exponential random variable with intensity parameter $Q_{0}(i, i)$. The probability of moving to state $j \neq i$ is $-Q_{0}(i, j) / Q_{0}(i, i)$.

\footnotetext{
${ }^{3}$ An intensity matrix has negative diagonal and non-negative off-diagonal entries. Each row sums up to zero.
} 
The model is fully determined if an initial state (or, more generally, an initial distribution $\pi_{0}:=\left(\mathbb{P}\left(Z_{0}=1\right), \mathbb{P}\left(Z_{0}=2\right), \ldots, \mathbb{P}\left(Z_{0}=M\right)\right)$ on the states $)$ is defined. The characteristic function of a regime switching model is given by (see, e.g., Buffington and Elliott [2002]; Elliott et al. [2005])

$$
\begin{aligned}
\phi_{t}(u) & :=\mathbb{E}\left[\exp \left(\boldsymbol{i} u\left(B_{t}-x\right)\right)\right] \\
& =\left\langle\exp \left(Q_{0} t+\left(\begin{array}{ccc}
\boldsymbol{i} u \mu_{1}-\frac{1}{2} \sigma_{1}^{2} u^{2} & 0 & \ldots \\
0 & \ldots & 0 \\
\ldots & 0 & \boldsymbol{i} u \mu_{M}-\frac{1}{2} \sigma_{M}^{2} u^{2}
\end{array}\right) t\right) \pi_{0}^{\prime}, \mathbf{1}\right\rangle,
\end{aligned}
$$

where $\exp (\cdot)$ denotes the matrix exponential function, ' transpose, 1 a vector of ones of appropriate size, and $\langle\cdot, \cdot\rangle$ the scalar product. The first-passage times on two constant barriers $b<B_{0}=x<a$ are defined as

$$
T_{a b}:= \begin{cases}\inf \left\{t \geq 0: B_{t} \notin(b, a)\right\}, & \text { if such a } t \text { exists, } \\ \infty, & \text { if } B_{t} \text { never hits the barriers. }\end{cases}
$$

Here, $T_{a b}$ is the first time the Brownian motion $B_{t}$ hits one of the two barriers $a$ and $b$. Further denote

$$
T_{a b}^{+}:=T_{a b}, \text { if } B_{T_{a b}} \geq a, \quad T_{a b}^{-}:=T_{a b}, \text { if } B_{T_{a b}} \leq b .
$$

The Laplace transform of the first-passage time is defined as

$$
\Psi_{a b}^{ \pm}(u):=\mathbb{E}\left[\exp \left(-u T_{a b}^{ \pm}\right)\right] .
$$

\section{Review of the matrix Wiener-Hopf factorization}

The rudiment of this work is the matrix Wiener-Hopf factorization as introduced by many authors, for example London et al. [1982], Kennedy and Williams [1990], Barlow et al. [1990], Rogers [1994], Asmussen [1995], and many others. A short review of the results is given in this section. The first-passage time problem of the Markov process $(B, Z)$ is closely linked to the up-crossing and down-crossing ladder processes $\overline{B_{t}}:=\max _{0 \leq s \leq t} B_{s}$ and $\underline{B_{t}}:=\min _{0 \leq s \leq t} B_{s}$. The ladder processes observe $B$ only when it is at its maximum or minimum, respectively. One can easily verify that $\overline{B_{t}}$ and $\underline{B_{t}}$ are again Markov processes on the same state space. Their generator matrices are linked to the so-called matrix Wiener-Hopf factorization $\left(Q_{+}, Q_{-}\right)$of the Markov process $(B, Z)$, see Definition 1.

\section{Definition 1 (Matrix Wiener-Hopf factorization)}

We denote the class of irreducible $M \times M$ generator matrices (non-negative off-diagonal entries and non-positive row sums) by $\mathcal{Q}_{M}$. A tuple $\left(Q_{+}, Q_{-}\right)$, where $Q_{+}, Q_{-} \in \mathcal{Q}_{M}$, is called a matrix Wiener-Hopf factorization of $(B, Z)$ if $\Xi\left(-Q_{+}\right)=\Xi\left(Q_{-}\right)=0, u>0$,

$$
\Xi(Q):=\frac{1}{2} \Sigma^{2} Q^{2}+V Q+Q_{0}-u I_{M}
$$

$\Sigma:=\operatorname{diag}\left(\sigma_{1}, \sigma_{2}, \ldots, \sigma_{M}\right), V:=\operatorname{diag}\left(\mu_{1}, \mu_{2}, \ldots, \mu_{M}\right)$, and $I_{M}$ is an $M \times M$ identity matrix. 


\section{Theorem 2 (First-passage times)}

(a) The matrix Wiener-Hopf factorization $\left(Q_{+}, Q_{-}\right)$from Definition 1 is unique.

(b) The Laplace transform of the first-passage time is $\Psi_{a b}^{ \pm}(u):=\pi_{0} \psi_{a b}^{ \pm}(u, x) \mathbf{1}$, where $\pi_{0} \in$ $\mathbb{R}^{1 \times M}$ is the initial distribution on the states and $\mathbf{1}$ a vector of ones of appropriate size. For two constant barriers $b<B_{0}=x<a$, we get in the single-barrier case

$$
\begin{aligned}
& \psi_{a,-\infty}^{+}(u, x):=\lim _{b \rightarrow-\infty} \psi_{a b}^{+}(u, x)=\exp \left(Q_{+}(a-x)\right), \\
& \psi_{\infty, b}^{-}(u, x):=\lim _{a \rightarrow \infty} \psi_{a b}^{-}(u, x)=\exp \left(Q_{-}(x-b)\right) .
\end{aligned}
$$

In the double barrier case ${ }^{4}$

$$
\begin{aligned}
& \psi_{a b}^{+}(u, x)=\left(\exp \left(Q_{+}(a-x)\right)-\exp \left(Q_{-}(x-b)\right) R_{+}\right) \sum_{k=0}^{\infty}\left(R_{-} R_{+}\right)^{k}, \\
& \psi_{a b}^{-}(u, x)=\left(\exp \left(Q_{-}(x-b)\right)-\exp \left(Q_{+}(a-x)\right) R_{-}\right) \sum_{k=0}^{\infty}\left(R_{+} R_{-}\right)^{k},
\end{aligned}
$$

where $R_{ \pm}:=\exp \left(Q_{ \pm}(a-b)\right)$ and $\exp (\cdot)$ denotes the matrix exponential.

\section{Proof}

Since $Q_{0}-u I_{M}$ is transient (its row sums are $-u<0$ ), (a) can be derived from Theorem 4.2(ii) in Jiang and Pistorius [2008]. For part (b), we refer to, for example, Rogers [1994] and Jiang and Pistorius [2008].

Similar results have been derived in the more general case of regime switching exponential jump-diffusion models, see, e.g., Jiang and Pistorius [2008]. The first-passage time problems then rest on solving matrix equations similar to those in Definition 1. Usually, this type of problem has to be solved numerically. The contribution of this work, however, is to present certain cases where one can obtain the matrix Wiener-Hopf factors $\left(Q_{+}, Q_{-}\right)$in closed-form, i.e. the case of 2 and 3 regimes or the case where $\mu_{Z_{t}} / \sigma_{Z_{t}}^{2}$ is regime-independent.

\section{First-passage time results}

First, we use Theorem 2 to recover known result for $M=1$ states, i.e. Brownian motion. Here, Equation (6) simplifies to $\frac{1}{2} \sigma_{1}^{2} \beta^{2}+\mu_{1} \beta=u$. The unique positive and negative root of this equation is $Q_{-}=\left(-\mu_{1}-\sqrt{\mu_{1}^{2}+2 u \sigma_{1}^{2}}\right) / \sigma_{1}^{2}$ and $-Q_{+}=\left(-\mu_{1}+\sqrt{\mu_{1}^{2}+2 u \sigma_{1}^{2}}\right) / \sigma_{1}^{2}$. From Theorem 2, using the identity $\sum_{k=0}^{\infty}\left(R_{-} R_{+}\right)^{k}=\left(I-R_{-} R_{+}\right)^{-1}$, we find that

$$
\Psi_{a b}^{+}(u)=\frac{\exp \left(Q_{+}(a-x)\right)-\exp \left(Q_{-}(x-b)\right) \exp \left(Q_{+}(a-b)\right)}{1-\exp \left(\left(Q_{+}+Q_{-}\right)(a-b)\right)}
$$

\footnotetext{
${ }^{4}$ Note that the identity $\sum_{k=0}^{\infty}\left(R_{-} R_{+}\right)^{k}=\left(I-R_{-} R_{+}\right)^{-1}$ might allow a faster computation; $\psi_{a b}^{ \pm}(u, x)$ can
} then be computed as the solution of a linear equation. 


$$
\begin{gathered}
=\exp \left(\frac{\mu_{1}}{\sigma_{1}^{2}}(a-x)\right) \frac{\sinh \left(\frac{\sqrt{\mu_{1}^{2}+2 \sigma_{1}^{2} u}}{\sigma_{1}^{2}}(x-b)\right)}{\sinh \left(\frac{\sqrt{\mu_{1}^{2}+2 \sigma_{1}^{2} u}}{\sigma_{1}^{2}}(a-b)\right)} \\
\Psi_{a,-\infty}^{+}(u)=\exp \left(Q_{+}(a-x)\right)=\exp \left(\frac{\mu_{1}(a-x)}{\sigma_{1}^{2}}\left(1-\sqrt{1+\frac{2 \sigma_{1}^{2} u}{\mu_{1}^{2}}}\right)\right) .
\end{gathered}
$$

Lemma 3 inverts the Laplace transforms in Equations (11)-(12) (see, for example, the inversion tables in Oberhettinger and Badii [1973], p. 295) to obtain the first-passage time probabilities of Brownian motion.

\section{Lemma 3 (Brownian motion: First-passage time probabilities)}

Consider a Brownian motion $B_{t}$ with drift $\mu_{1}$ and volatility $\sigma_{1}>0$ and $T>0$.

(a) The first-passage time probability on a single-barrier $a>x=B_{0}$ is given by

$$
\mathbb{P}\left(T_{a,-\infty} \leq T\right)=\Phi\left(\frac{(x-a)+\mu_{1} T}{\sigma_{1} \sqrt{T}}\right)+\exp \left(\frac{2 \mu_{1}(a-x)}{\sigma_{1}^{2}}\right) \Phi\left(\frac{(x-a)-\mu_{1} T}{\sigma_{1} \sqrt{T}}\right),
$$

where $\Phi(\cdot)$ denotes the standard normal cumulative density function.

(b) Consider two barriers $b<x=B_{0}<a$. If $\mu_{1}=0$, the double-barrier first-passage time probability is

$$
\mathbb{P}\left(T_{a b}^{+} \leq T\right)=\frac{x-b}{a-b}+K_{T}^{\infty}(b-x),
$$

for $\mu_{1} \neq 0$

$$
\mathbb{P}\left(T_{a b}^{+} \leq T\right)=\frac{\exp \left(-\frac{2 \mu_{1}(b-x)}{\sigma_{1}^{2}}\right)-1}{\exp \left(-\frac{2 \mu_{1}(b-x)}{\sigma_{1}^{2}}\right)-\exp \left(-\frac{2 \mu_{1}(a-x)}{\sigma_{1}^{2}}\right)}+\exp \left(\frac{\mu_{1}(a-x)}{\sigma_{1}^{2}}\right) K_{T}^{\infty}(b-x),
$$

$$
\text { where } K_{T}^{N}(k):=\frac{\sigma_{1}^{2} \pi}{(a-b)^{2}} \sum_{n=1}^{N} \frac{n(-1)^{n+1}}{\frac{\mu_{1}^{2}}{2 \sigma_{1}^{2}}+\frac{\sigma_{1}^{2} n^{2} \pi^{2}}{2(a-b)^{2}}} \exp \left(-\left(\frac{\mu_{1}^{2}}{2 \sigma_{1}^{2}}+\frac{\sigma_{1}^{2} n^{2} \pi^{2}}{2(a-b)^{2}}\right) T\right) \sin \left(\frac{n \pi k}{a-b}\right) \text {. }
$$

\section{Proof}

Part (a) is the cumulative distribution function of an inverse Gaussian distribution and thus the Laplace inverse of Equation (12), see, e.g., Folks and Chhikara [1978]. Part (b) follows by Laplace inversion and integration on $(0, T)$, see, e.g., Hieber and Scherer [2012] for more details. A different (but similar) approach using renewal-type equations is presented in Darling and Siegert [1953]. Note that the expression in Darling and Siegert [1953] contains two typos: $\pi^{2}$ has to be replaced by $\pi$ and $(-1)^{n}$ by $(-1)^{n+1}$.

The next step is the 2-state model $(M=2)$. The solution depends on the roots of the so-called Cramér-Lundberg equation given by

$$
\left(\frac{1}{2} \sigma_{1}^{2} \beta^{2}+\mu_{1} \beta+q_{11}-u\right)\left(\frac{1}{2} \sigma_{2}^{2} \beta^{2}+\mu_{2} \beta+q_{22}-u\right)-q_{11} q_{22}=0,
$$


where $q_{11}:=Q_{0}(1,1)$ and $q_{22}:=Q_{0}(2,2)$. This equation has 4 unique real roots $-\infty<\beta_{1, u}<$ $\beta_{2, u}<0<\beta_{3, u}<\beta_{4, u}<\infty$ (see, for example, Guo [2001b] for a proof). Those roots are available in closed-form, see, for example, Abramowitz and Stegun [1965], p. $17 \mathrm{f}$.

\section{Main Theorem 4 (2-state model: Matrix Wiener-Hopf factorization)}

Consider the regime switching model as defined in Equation (1) with $M=2$ states and $q_{11} q_{22} \neq$ 0 .

(a) The matrix Wiener-Hopf factorization $\left(Q_{+}, Q_{-}\right)$is given by

$$
\begin{aligned}
& Q_{+}=\left(\begin{array}{cc}
\frac{-\beta_{3, u} \beta_{4, u}+\frac{2\left(q_{11}-u\right)}{\sigma_{1}^{2}}}{\beta_{3, u}+\beta_{4, u}+\frac{2 \mu_{1}}{\sigma_{1}^{2}}} & \frac{-\frac{2 q_{11}}{\sigma_{1}^{2}}}{\beta_{3, u}+\beta_{4, u}+\frac{2 \mu_{1}}{\sigma_{2}^{2}}} \\
\frac{-\frac{2 q_{22}}{\sigma_{2}^{2}}}{\beta_{3, u}+\beta_{4, u}+\frac{2 \mu_{2}}{\sigma_{2}^{2}}} & \frac{-\beta_{3, u} \beta_{4, u}+\frac{2\left(q_{22}-u\right)}{\sigma_{2}^{2}}}{\beta_{3, u}+\beta_{4, u}+\frac{2 \mu_{2}}{\sigma_{2}^{2}}}
\end{array}\right), \\
& Q_{-}=\left(\begin{array}{cc}
\frac{\beta_{1, u} \beta_{2, u}-\frac{2\left(q_{11}-u\right)}{\sigma_{1}^{2}}}{\beta_{1, u}+\beta_{2, u}+\frac{2 \mu_{1}}{\sigma_{1}^{2}}} & \frac{2 q_{11}}{\sigma_{1}^{2}} \\
\frac{\beta_{1, u}+\beta_{2, u}+\frac{2 \mu_{1}}{\sigma_{1}^{2}}}{\sigma_{2}^{2}} & \frac{\beta_{1, u} \beta_{2, u}-\frac{2\left(q_{22}-u\right)}{\sigma_{2}^{2}}}{\beta_{1, u}+\beta_{2, u}+\frac{2 \mu_{2}}{\sigma_{2}^{2}}} \\
\beta_{1, u}+\beta_{2, u}+\frac{2 \mu_{2}}{\sigma_{2}^{2}}
\end{array}\right),
\end{aligned}
$$

where $Q_{0}:=\left[q_{11}-q_{11} ;-q_{22} q_{22}\right]$ and $-\infty<\beta_{1, u}<\beta_{2, u}<0<\beta_{3, u}<\beta_{4, u}<\infty$ are the roots of Equation (16).

(b) The matrix exponentials of $\left(Q_{+}, Q_{-}\right)$are, for $k \in \mathbb{R}^{+}$, given by

$$
\begin{aligned}
& \exp \left(Q_{+} k\right)=\frac{\beta_{3, u} e^{-\beta_{4, u} k}-\beta_{4, u} e^{-\beta_{3, u} k}}{\beta_{3, u}-\beta_{4, u}}\left(\begin{array}{ll}
1 & 0 \\
0 & 1
\end{array}\right)+\frac{e^{-\beta_{3, u} k}-e^{-\beta_{4, u} k}}{\beta_{3, u}-\beta_{4, u}} Q_{+}, \\
& \exp \left(Q_{-} k\right)=\frac{\beta_{1, u} e^{\beta_{2, u} k}-\beta_{2, u} e^{\beta_{1, u} k}}{\beta_{1, u}-\beta_{2, u}}\left(\begin{array}{ll}
1 & 0 \\
0 & 1
\end{array}\right)+\frac{e^{\beta_{1, u} k}-e^{\beta_{2, u} k}}{\beta_{1, u}-\beta_{2, u}} Q_{-} .
\end{aligned}
$$

\section{Proof}

For part (a), one has to solve Equation (6), i.e. find the unique solutions $Q_{-} \in \mathcal{Q}_{2}$ and $-Q_{+} \in \mathcal{Q}_{2}$ of

$$
\frac{1}{2}\left(\begin{array}{cc}
\sigma_{1}^{2} & 0 \\
0 & \sigma_{2}^{2}
\end{array}\right) Q^{2}+\left(\begin{array}{cc}
\mu_{1} & 0 \\
0 & \mu_{2}
\end{array}\right) Q+\left(\begin{array}{cc}
q_{11}-u & -q_{11} \\
-q_{22} & q_{22}-u
\end{array}\right)=\left(\begin{array}{cc}
0 & 0 \\
0 & 0
\end{array}\right)
$$

Parameterizing $Q_{-}:=\left[a_{11} a_{12} ; a_{21} a_{22}\right]$, this is a set of 4 quadratic equations with 4 unknowns. The top left entry in Equation (19) is $\frac{1}{2} \sigma_{1}^{2}\left(a_{11}^{2}+a_{12} a_{21}\right)+a_{11} \mu_{1}+q_{11}-u=0$ which is equivalent to

$$
a_{12} a_{21}=-\frac{2\left(q_{11}-u\right)}{\sigma_{1}^{2}}-\frac{2 \mu_{1}}{\sigma_{1}^{2}} a_{11}-a_{11}^{2}
$$

Furthermore, $\frac{1}{2} \sigma_{1}^{2} a_{12}\left(a_{11}+a_{22}\right)+a_{12} \mu_{1}-q_{11}=0$, which is equivalent to

$$
a_{12}=\frac{2 q_{11}}{\sigma_{1}^{2}\left(a_{11}+a_{22}+\frac{2 \mu_{1}}{\sigma_{1}^{2}}\right)} .
$$


Since $Q_{-} \in \mathcal{Q}_{2}$, there have to be two eigenvalues $\lambda_{1} \leq \lambda_{2}<0$ and corresponding eigenvectors $v_{1} \in \mathbb{R}^{2 \times 1}$ and $v_{2} \in \mathbb{R}^{2 \times 1}$. If Equation (19) holds, we find that

$$
\left[\frac{1}{2}\left(\begin{array}{cc}
\sigma_{1}^{2} & 0 \\
0 & \sigma_{2}^{2}
\end{array}\right) \lambda_{i}^{2}+\left(\begin{array}{cc}
\mu_{1} & 0 \\
0 & \mu_{2}
\end{array}\right) \lambda_{i}+\left(\begin{array}{cc}
q_{11}-u & -q_{11} \\
-q_{22} & q_{22}-u
\end{array}\right)\right] v_{i}=\left(\begin{array}{l}
0 \\
0
\end{array}\right), \quad i=1,2 .
$$

The latter expression is solved if we choose $\lambda_{1}=\beta_{1, u}, v_{1}=\left(\sigma_{2}^{2} \beta_{1, u}^{2} / 2+\mu_{2} \beta_{1, u}+q_{22}-u ; q_{22}\right)$ and $\lambda_{2}=\beta_{2, u}, v_{2}=\left(q_{11} ; \sigma_{1}^{2} \beta_{2, u}^{2} / 2+\mu_{1} \beta_{2, u}+q_{11}-u\right)$. Using basic identities from the characteristic polynomial, we can at once conclude that $a_{11}+a_{22}=\beta_{1, u}+\beta_{2, u}$ and $\operatorname{det}\left(Q_{-}\right)=$ $\beta_{1, u} \beta_{2, u}$. Then, we obtain $a_{12}$ from Equation (21). From the determinant of $Q_{-}$, we find that $a_{11}\left(\beta_{1, u}+\beta_{2, u}-a_{11}\right)-a_{12} a_{21}=\beta_{1, u} \beta_{2, u}$ which is, with the help of Equation (20), equivalent to

$$
a_{11}=\frac{\beta_{1, u} \beta_{2, u}-\frac{2\left(q_{11}-u\right)}{\sigma_{1}^{2}}}{\beta_{1, u}+\beta_{2, u}+\frac{2 \mu_{1}}{\sigma_{1}^{2}}} .
$$

Similarly, exchanging the triplets $\left(\mu_{1}, \sigma_{1}, q_{11}\right)$ and $\left(\mu_{2}, \sigma_{2}, q_{22}\right)$ in Equations (20) and (21), the expressions for $a_{21}$ and $a_{22}$ are derived. The resulting matrix $Q_{-}$is a sub-generator matrix since its determinant $\beta_{1, u} \beta_{2, u}$ is positive, its row sums are non-positive, and its off-diagonal entries are non-negative. By symmetry (replacing $\mu_{1}$ by $-\mu_{1}, \mu_{2}$ by $-\mu_{2}, \beta_{1, u}$ by $-\beta_{4, u}$, and $\beta_{2, u}$ by $\left.-\beta_{3, u}\right)^{4}$, the same considerations lead to the given representation of $Q_{+}$.

Knowing that $\beta_{1, u}<\beta_{2, u}<0$ are the two eigenvalues of $Q_{-}$, part (b) is straightforward.

The Laplace transforms of the first-passage times are then a straightforward implication of Theorems 2 and 4 . Corollary 5 presents the Laplace transforms of the single barrier firstpassage times. This result corrects Guo [2001a], see also Hieber [2013].

\section{Corollary 5 (2-state model: First-passage time)}

Consider the regime switching model as defined in Equation (1) with $M=2$ states and $q_{11} q_{22} \neq$ 0 . For an initial distribution on the states $\pi_{0}=(\bar{\pi}, 1-\bar{\pi}):=\left(\mathbb{P}\left(Z_{0}=1\right), \mathbb{P}\left(Z_{0}=2\right)\right)$, the Laplace transform of the first passage time on an upper barrier $a>B_{0}=x$, respectively $a$ lower barrier $b<B_{0}=x$, is given by

$$
\begin{aligned}
& \Psi_{a,-\infty}^{+}(u)=\frac{\beta_{3, u} e^{-\beta_{4, u}(a-x)}-\beta_{4, u} e^{-\beta_{3, u}(a-x)}}{\beta_{3, u}-\beta_{4, u}} \\
& -\frac{e^{-\beta_{4, u}(a-x)}-e^{-\beta_{3, u}(a-x)}}{\beta_{3, u}-\beta_{4, u}}\left(\bar{\pi} \frac{\beta_{3, u} \beta_{4, u}+\frac{2 u}{\sigma_{1}^{2}}}{\beta_{3, u}+\beta_{4, u}+\frac{2 \mu_{1}}{\sigma_{1}^{2}}}+(1-\bar{\pi}) \frac{\beta_{3, u} \beta_{4, u}+\frac{2 u}{\sigma_{2}^{2}}}{\beta_{3, u}+\beta_{4, u}+\frac{2 \mu_{2}}{\sigma_{2}^{2}}}\right), \\
& \Psi_{\infty, b}^{-}(u)=\frac{\beta_{1, u} e^{\beta_{2, u}(x-b)}-\beta_{2, u} e^{\beta_{1, u}(x-b)}}{\beta_{1, u}-\beta_{2, u}} \\
& +\frac{e^{\beta_{1, u}(x-b)}-e^{\beta_{2, u}(x-b)}}{\beta_{1, u}-\beta_{2, u}}\left(\bar{\pi} \frac{\beta_{1, u} \beta_{2, u}+\frac{2 u}{\sigma_{1}^{2}}}{\beta_{1, u}+\beta_{2, u}+\frac{2 \mu_{1}}{\sigma_{1}^{2}}}+(1-\bar{\pi}) \frac{\beta_{1, u} \beta_{2, u}+\frac{2 u}{\sigma_{2}^{2}}}{\beta_{1, u}+\beta_{2, u}+\frac{2 \mu_{2}}{\sigma_{2}^{2}}}\right),
\end{aligned}
$$

where $-\infty<\beta_{1, u}<\beta_{2, u}<0<\beta_{3, u}<\beta_{4, u}<\infty$ are the roots of Equation (16).

\footnotetext{
${ }^{4}$ Note that the transformation $\mu_{1} \mapsto-\mu_{1}, \mu_{2} \mapsto-\mu_{2}$ changes the sign of the roots of Equation (16).
} 


\section{Proof}

If we set $k=(x-b)$ in Theorem 4 and apply the results from Theorem 2 we can conclude that

$$
\begin{aligned}
\Psi_{\infty, b}^{-}(u):= & \pi_{0} \exp \left(Q_{-}(x-b)\right)\left(\begin{array}{l}
1 \\
1
\end{array}\right) \\
= & \pi_{0}\left(\frac{\beta_{1, u} e^{\beta_{2, u}(x-b)}-\beta_{2, u} e^{\beta_{1, u}(x-b)}}{\beta_{1, u}-\beta_{2, u}}\left(\begin{array}{ll}
1 & 0 \\
0 & 1
\end{array}\right)+\frac{e^{\beta_{1, u}(x-b)}-e^{\beta_{2, u}(x-b)}}{\beta_{1, u}-\beta_{2, u}} Q_{-}\right)\left(\begin{array}{l}
1 \\
1
\end{array}\right) \\
= & \frac{\beta_{1, u} e^{\beta_{2, u}(x-b)}-\beta_{2, u} e^{\beta_{1, u}(x-b)}}{\beta_{1, u}-\beta_{2, u}} \\
& \quad+\frac{e^{\beta_{1, u}(x-b)}-e^{\beta_{2, u}(x-b)}}{\beta_{1, u}-\beta_{2, u}}\left(\bar{\pi} \frac{\beta_{1, u} \beta_{2, u}+\frac{2 u}{\sigma_{1}^{2}}}{\beta_{1, u}+\beta_{2, u}+\frac{2 \mu_{1}}{\sigma_{1}^{2}}}+(1-\bar{\pi}) \frac{\beta_{1, u} \beta_{2, u}+\frac{2 u}{\sigma_{2}^{2}}}{\beta_{1, u}+\beta_{2, u}+\frac{2 \mu_{2}}{\sigma_{2}^{2}}}\right) .
\end{aligned}
$$

Note that, in contrast to Guo [2001a], $\mu_{1}$ and $\mu_{2}$ can also take positive values.

In the 3-state model, the matrix Wiener-Hopf factorization can - for $\beta \in \mathbb{C}$ - be expressed by the determinant of $\frac{1}{2} \Sigma^{2} \beta^{2}+V \beta+Q_{0}-u I_{M}$. We refer to this 6-degree polynomial as Cramér-Lundberg equation, for $g_{i}(\beta):=\sigma_{i}^{2} \beta^{2} / 2+\mu_{i} \beta+q_{i i}-u, i \in\{1,2,3\}$, given by

$$
g_{1}(\beta) g_{2}(\beta) g_{3}(\beta)-q_{23} q_{32} g_{1}(\beta)-q_{13} q_{31} g_{2}(\beta)-q_{21} q_{12} g_{3}(\beta)+q_{13} q_{21} q_{32}+q_{12} q_{23} q_{31}=0,
$$

where $-\infty<\operatorname{Re}\left(\beta_{1, u}\right) \leq \operatorname{Re}\left(\beta_{2, u}\right) \leq \operatorname{Re}\left(\beta_{3, u}\right)<0<\operatorname{Re}\left(\beta_{4, u}\right) \leq \operatorname{Re}\left(\beta_{5, u}\right) \leq \operatorname{Re}\left(\beta_{6, u}\right)<\infty$ (see, e.g., Barlow et al. [1990] for a proof). In practical applications, it turns out to be sufficient to consider distinct roots only (see, e.g., Kalbfleisch et al. [1983], Rogers and Shi [1994]):

\section{Assumption 1 (Uniqueness of roots)}

The roots of Equation (22) are distinct, i.e. $-\infty<\operatorname{Re}\left(\beta_{1, u}\right)<\operatorname{Re}\left(\beta_{2, u}\right)<\operatorname{Re}\left(\beta_{3, u}\right)<0<$ $\operatorname{Re}\left(\beta_{4, u}\right)<\operatorname{Re}\left(\beta_{5, u}\right)<\operatorname{Re}\left(\beta_{6, u}\right)<\infty$.

The latter assumption can rather easily be relaxed, but leads to unnecessary complicated expressions for the matrix Wiener-Hopf factorization. Theorem 6 presents the matrix WienerHopf factorization in the 3 -state model.

\section{Main Theorem 6 (3-state model: Matrix Wiener-Hopf factorization)}

Consider the regime switching model as defined in Equation (1) with irreducible intensity matrix $Q_{0}$ and $M=3$ states. Assume that Assumption 1 holds. The matrix Wiener-Hopf factorization $\left(Q_{+}, Q_{-}\right)$is given by

$$
\begin{aligned}
& Q_{+}=-Z_{+}\left(\begin{array}{ccc}
\beta_{4, u} & 0 & 0 \\
0 & \beta_{5, u} & 0 \\
0 & 0 & \beta_{6, u}
\end{array}\right) Z_{+}^{-1}, \quad Q_{-}=Z_{-}\left(\begin{array}{ccc}
\beta_{1, u} & 0 & 0 \\
0 & \beta_{2, u} 0 & \\
0 & 0 & \beta_{3, u}
\end{array}\right) Z_{-}^{-1}, \\
& v_{i}:=\left(\begin{array}{c}
g_{1}\left(\beta_{i, u}\right) \\
q_{12} \\
q_{13}
\end{array}\right) \bigotimes\left(\begin{array}{c}
q_{21} \\
g_{2}\left(\beta_{i, u}\right) \\
q_{23}
\end{array}\right),{ }^{6}
\end{aligned}
$$


where $Z_{-}:=\left[v_{1} v_{2} v_{3}\right], Z_{+}:=\left[v_{4} v_{5} v_{6}\right], Q_{0}:=\left[q_{11} q_{12} q_{13} ; q_{21} q_{22} q_{23} ; q_{31} q_{32} q_{33}\right], \bigotimes$ denotes the cross product ${ }^{5}, g_{1}(\cdot)$ and $g_{2}(\cdot)$ are defined in Equation (22), and $\beta_{1, u}, \beta_{2, u}, \beta_{3, u}, \beta_{4, u}$, $\beta_{5, u}, \beta_{6, u}$ are the roots of Equation (22).

\section{Proof}

Again, we have to search for solutions $\left(Q_{+}, Q_{-}\right) \in\left(\mathcal{Q}_{3}, \mathcal{Q}_{3}\right)$ of

$$
\Xi(Q):=\frac{1}{2} \Sigma^{2} Q^{2}+V Q+Q_{0}-u I_{M}=0 .
$$

For a given pair of eigenvalues and eigenvectors $\left(\beta_{i, u}, v_{i}\right) \in\left(\mathbb{C}, \mathbb{C}^{3 \times 1}\right), i \in\{1,2, \ldots, 6\}$, we find that $\Xi\left(\beta_{i, u}\right) v_{i}=0$. Due to Assumption 1, there has to be one (unique) vector $v_{i}$ for every $i \in\{1,2, \ldots, 6\}$ that fulfills $\Xi\left(\beta_{i, u}\right) v_{i}=0$, i.e. a (unique) eigenvector of $\Xi\left(\beta_{i, u}\right)$ with eigenvalue 0 .

As we already noted in the 2-state case, $\beta_{i, u}, v_{i}, i \in\{4,5,6\}$ are the eigenvalues, respectively eigenvectors, of the (diagonalizable) matrix $Q_{+}$. Basic algebraic theory on the diagonalization of matrices then yields

$$
Q_{+}=-Z_{+}\left(\begin{array}{ccc}
\beta_{4, u} & 0 & 0 \\
0 & \beta_{5, u} & 0 \\
0 & 0 & \beta_{6, u}
\end{array}\right) Z_{+}^{-1}
$$

where $Z_{+}:=\left[v_{4} v_{5} v_{6}\right]$. Similar arguments lead to the expression for $Q_{-}$.

In the $M$-state case, we want to refrain from complicated semi-analytical expressions for the matrix Wiener-Hopf factors and instead present an efficient numerical algorithm by Rogers and Shi [1994], Jobert and Rogers [2006]. The basic idea of this algorithm is the fact that for any eigenvalue/eigenvector pair $\left(\beta_{i, u}, v_{i}\right) \in\left(\mathbb{C}, \mathbb{C}^{M \times 1}\right), i=\{1,2, \ldots, 2 M\}$, the (quadratic) eigenvalue problem

$$
\left[\frac{1}{2} \Sigma^{2} Q^{2}+V Q+\left(Q_{0}-u I_{M}\right)\right] v_{i}=\left[\beta_{i, u}^{2}+2 \Sigma^{-2} V \beta_{i, u}+2 \Sigma^{-2}\left(Q_{0}-u I_{M}\right)\right] v_{i}=0
$$

can be transformed to

$$
\begin{aligned}
w_{i} & =\beta_{i, u} v_{i}, \\
-2 \Sigma^{-2}\left(Q_{0}-u I_{M}\right) v_{i}-2 \Sigma^{-2} V w_{i} & =\beta_{i, u} w_{i},
\end{aligned}
$$

where $w_{i} \in \mathbb{C}^{M \times 1}$. This yields the linear eigenvalue problem

$$
\left(\begin{array}{cc}
0 & I_{M} \\
-2 \Sigma^{-2}\left(Q_{0}-u I_{M}\right) & -2 \Sigma^{-2} V
\end{array}\right)\left(\begin{array}{c}
v_{i} \\
w_{i}
\end{array}\right)=\beta_{i, u}\left(\begin{array}{c}
v_{i} \\
w_{i}
\end{array}\right)
$$

This eigenvalue problem has obviously $2 M$ solutions. If $u>0$, half of the eigenvalues $\beta_{i, u}$ lie in the left open half plane, the other half in the right open half plane, see Barlow et al. [1990]. Algorithm 1 presents the necessary steps to obtain the matrix Wiener-Hopf factors $\left(Q_{+}, Q_{-}\right)$ numerically.

\footnotetext{
${ }^{5}$ The cross product is defined as $\left[a_{1} ; a_{2} ; a_{3}\right] \otimes\left[b_{1} ; b_{2} ; b_{3}\right]:=\left[a_{2} b_{3}-a_{3} b_{2} ; a_{3} b_{1}-a_{1} b_{3} ; a_{1} b_{2}-a_{2} b_{1}\right]$.

${ }^{6}$ In some rare or artificial cases (e.g. $\mu_{1}=\mu_{2}=\mu_{3}, \sigma_{1}=\sigma_{2}=\sigma_{3}$ ), this cross product might be 0 . If this is the case $v_{i}$ is instead set to $v_{i}:=\left(q_{21} g_{2}\left(\beta_{i, u}\right) q_{23}\right) \otimes\left(q_{31} q_{32} g_{3}\left(\beta_{i, u}\right)\right)$. Due to the fact that the roots $\beta_{i, u}$ are distinct, one of these cross products has to be non-zero.
} 


\section{Algorithm 1 (Rogers [1994]: Matrix Wiener-Hopf factorization)}

This algorithm computes the matrix Wiener-Hopf factors $\left(Q_{+}, Q_{-}\right)$by diagonalization. As an input, the matrices $\Sigma:=\operatorname{diag}\left(\sigma_{1}, \sigma_{2}, \ldots, \sigma_{M}\right), V:=\operatorname{diag}\left(\mu_{1}, \mu_{2}, \ldots, \mu_{M}\right)$, the intensity matrix $Q_{0}$, and $u>0$ are needed.

1. Determine the eigenvalue/eigenvector pairs $\left(\beta_{i, u}, v_{i}\right) \in\left(\mathbb{C}, \mathbb{C}^{M \times 1}\right), i=\{1,2, \ldots, 2 M\}$, of

$$
\left(\begin{array}{cc}
0 & I_{M} \\
-2 \Sigma^{-2}\left(Q_{0}-u I_{M}\right) & -2 \Sigma^{-2} V
\end{array}\right)\left(\begin{array}{c}
v_{i} \\
w_{i}
\end{array}\right)=\beta_{i, u}\left(\begin{array}{c}
v_{i} \\
w_{i}
\end{array}\right)
$$

and sort them according to their real value $\left(\operatorname{Re}\left(\beta_{1, u}\right)<\operatorname{Re}\left(\beta_{2, u}\right)<\ldots<\operatorname{Re}\left(\beta_{2 M, u}\right)\right)$.

2. $\operatorname{Set}^{7} Z_{-}:=\left[\begin{array}{lll}v_{1} & \ldots & v_{M}\end{array}\right]$ and $Z_{+}:=\left[v_{M+1} \ldots v_{2 M}\right]$ to get

$$
Q_{+}=-Z_{+}\left(\begin{array}{ccc}
\beta_{M+1, u} & 0 & 0 \\
0 & \ldots & 0 \\
0 & 0 & \beta_{2 M, u}
\end{array}\right) Z_{+}^{-1}, \quad Q_{-}=Z_{-}\left(\begin{array}{ccc}
\beta_{1, u} & 0 & 0 \\
0 & \ldots & 0 \\
0 & 0 & \beta_{M, u}
\end{array}\right) Z_{-}^{-1} .
$$

However, for a regime switching model with more than three states over-fitting may occur in some cases. That is why it might make sense to restrict the parameter space. In the following, we assume that the quotient $\mu_{Z_{t}} / \sigma_{Z_{t}}^{2}$ is constant over all states. The matrix Wiener-Hopf factorizaton is then given in closed-form, see Theorem 7 .

\section{Main Theorem $7\left(\mu_{Z_{t}} / \sigma_{Z_{t}}^{2}\right.$ constant: Matrix Wiener-Hopf factorization)}

Consider the regime switching model (1) with $\mu_{1} / \sigma_{1}^{2}=\mu_{2} / \sigma_{2}^{2}=\ldots=\mu_{M} / \sigma_{M}^{2}=: c \in \mathbb{R}$.

(a) The matrix Wiener-Hopf factorization $\left(Q_{+}, Q_{-}\right)$is given by

$$
Q_{ \pm}= \pm c I_{M}-\sqrt{c^{2} I_{M}-2 \Sigma^{-2}\left(Q_{0}-u I_{M}\right)} .
$$

(b) The Laplace transform of the single barrier first-passage time on an upper barrier a > $B_{0}=x$ is then given by

$$
\psi_{a,-\infty}(u)=\exp \left(Q_{+}(a-x)\right)=\exp \left(c(a-x)\left(I_{M}-\sqrt{I_{M}+\frac{2 \Sigma^{-2}\left(Q_{0}-u I_{M}\right)}{c^{2}}}\right)\right) .
$$

\section{Proof}

For $c:=\mu_{1} / \sigma_{1}^{2} \in \mathbb{R}$, we conclude from Equation (6) that

$0=2 \Sigma^{-2} \Xi(Q):=Q^{2}+2 \Sigma^{-2} V Q+2 \Sigma^{-2}\left(Q_{0}-u I_{M}\right)=\left(Q+c I_{M}\right)^{2}-c^{2} I_{M}+2 \Sigma^{-2}\left(Q_{0}-u I_{M}\right)$,

From this, we immediately obtain

$$
Q_{-}=-c I_{M}-\sqrt{c^{2} I_{M}-2 \Sigma^{-2}\left(Q_{0}-u I_{M}\right)}
$$

\footnotetext{
${ }^{7}$ This contains the implicit assumption that the eigenvectors $v_{i}$ form a basis, an assumption that turned out to be sufficient in practical applications (see, e.g., Rogers and Shi [1994] or Assumption 1). It is possible to construct artificial examples where such a basis does not exist. The following steps of Algorithm 1 can then be modified using a basis of Jordan vectors.
} 


$$
Q_{+}=-\left(-c I_{M}+\sqrt{c^{2} I_{M}-2 \Sigma^{-2}\left(Q_{0}-u I_{M}\right)}\right) .
$$

Part (b) is a straightforward corollary to Theorem 2.

Part (b) in Theorem 7 already indicates that the first-passage time problem in Theorem 7 is closely linked to the case of Brownian motion. Example 8 and Lemma 9 establish the connection.

\section{Example 8 (Bacterial growth)}

It is commonly accepted that there is a positive impact of temperatures on bacterial growth, see, for example, Ratkowsky et al. [1982]. We assume a very simplistic relationship and consider bacteria whose growth rate follows a regime switching model: If the temperature is between 0 and 45 degrees Celsius, the growth rate is described by a Brownian motion with parameters $\left(\mu_{1}, \sigma_{1}\right)=(10 \%, 5 \%)$; if it rises above 45 degrees Celsius, it quadruples to $\left(\mu_{2}, \sigma_{2}\right)=(40 \%, 10 \%)$. Then, the quotient $\mu_{Z_{t}} / \sigma_{Z_{t}}^{2}$ is constant, i.e. $\mu_{1} / \sigma_{1}^{2}=\mu_{2} / \sigma_{2}^{2}=40$.

There exists another - slightly different - viewpoint on Example 8: Assume that the growth rate is constant at $\left(\mu_{1}, \sigma_{1}\right)=(10 \%, 5 \%)$, but as soon as the temperature exceeds 45 degrees Celsius, we get the impression that time runs $\mu_{2} / \mu_{1}=4$ times faster than before. Time returns to the original pace if we return to temperatures below 45 degrees Celsius. What we found is a representation of the restricted model $\left(\mu_{1} / \sigma_{1}^{2}=\mu_{2} / \sigma_{2}^{2}=\ldots=\mu_{M} / \sigma_{M}^{2}=: c\right)$ as a time-changed Brownian motion $\left(\mu=\mu_{1}, \sigma=\sigma_{1}\right) B_{\Lambda_{T}}$. Lemma 9 gives a formal proof.

\section{Lemma 9 (Time-change representation)}

Consider the regime switching model (1) with $\mu_{1} / \sigma_{1}^{2}=\mu_{2} / \sigma_{2}^{2}=\ldots=\mu_{M} / \sigma_{M}^{2}=: c \in \mathbb{R}$ and initial distribution on the states $\pi_{0}:=\left(\mathbb{P}\left(Z_{0}=1\right), \mathbb{P}\left(Z_{0}=2\right), \ldots, \mathbb{P}\left(Z_{0}=M\right)\right)$. Then, the model can be represented as a time-changed Brownian motion $B_{\Lambda_{T}}$, where the time change $\left\{\Lambda_{t}\right\}_{t \geq 0}$ is independent of $B$. The Laplace transform of the time change $\Lambda_{T}$ is given by

$$
\vartheta_{T}(u):=\mathbb{E}\left[\exp \left(-u \Lambda_{T}\right)\right]=\left\langle\exp \left(Q_{0} T-\frac{u}{\sigma_{1}^{2}}\left(\begin{array}{ccc}
\sigma_{1}^{2} & 0 & \ldots \\
0 & \ldots & 0 \\
\ldots & 0 & \sigma_{M}^{2}
\end{array}\right) T\right) \pi_{0}^{\prime}, \mathbf{1}\right\rangle,
$$

where $\exp (\cdot)$ denotes the matrix exponential function, ${ }^{\prime}$ transpose, and $\langle\cdot, \cdot\rangle$ the scalar product.

\section{Proof}

From Equation (2), we conclude that

$$
\begin{aligned}
\phi_{T}(u) & =\left\langle\exp \left(Q_{0} T+\left(\begin{array}{ccc}
\boldsymbol{i} u \mu_{1}-\frac{1}{2} \sigma_{1}^{2} u^{2} & 0 & \ldots \\
0 & \ldots & 0 \\
\ldots & 0 & \boldsymbol{i} u \mu_{M}-\frac{1}{2} \sigma_{M}^{2} u^{2}
\end{array}\right) T\right) \pi_{0}^{\prime}, \mathbf{1}\right\rangle \\
& =\left\langle\exp \left(Q_{0} T+\frac{\boldsymbol{i} u \mu_{1}-\frac{1}{2} \sigma_{1}^{2} u^{2}}{\sigma_{1}^{2}}\left(\begin{array}{ccc}
\sigma_{1}^{2} & 0 & \ldots \\
0 & \ldots & 0 \\
\ldots & 0 & \sigma_{M}^{2}
\end{array}\right) T\right) \pi_{0}^{\prime}, \mathbf{1}\right\rangle=\vartheta_{T}\left(-\left(\boldsymbol{i} u \mu_{1}-\sigma_{1}^{2} u^{2} / 2\right)\right),
\end{aligned}
$$

where $i u \mu_{1}-\frac{1}{2} \sigma_{1}^{2} u^{2}$ is the characteristic exponent of a normal distribution. From $\phi_{T}(u)=$ $\vartheta_{T}\left(-\left(\boldsymbol{i} u \mu_{1}-\sigma_{1}^{2} u^{2} / 2\right)\right)$, we can conclude that the regime switching model can be represented as a time-changed Brownian motion, where the Laplace transform of the time change is $\vartheta_{T}(u)$. 
For time-changed Brownian motion, the Laplace transform of the first-passage time was derived by, e.g., Hurd [2009]. For the double barrier first-passage time, this Laplace transform can even be inverted to obtain a rapidly converging infinite series, see Hieber and Scherer [2012]. Theorem 10 applies those results to regime switching models. This extends Lemma 3.

Main Theorem 10 (M-state model, $\mu_{Z_{t}} / \sigma_{Z_{t}}^{2}$ constant: First-passage probabilities) Consider the regime switching model (1) with $\mu_{1} / \sigma_{1}^{2}=\mu_{2} / \sigma_{2}^{2}=\ldots=\mu_{M} / \sigma_{M}^{2}=: c \in \mathbb{R}$ and initial distribution on the states $\pi_{0}:=\left(\mathbb{P}\left(Z_{0}=1\right), \mathbb{P}\left(Z_{0}=2\right), \ldots, \mathbb{P}\left(Z_{0}=M\right)\right)$.

(a) The first-passage time probability on a barrier $a>x=B_{0}$ is given by

$$
\begin{aligned}
& \mathbb{P}\left(T_{a,-\infty} \leq T\right)=\frac{1+\exp \left(\frac{2 \mu_{1}(a-x)}{\sigma_{1}^{2}}\right)}{2} \\
& \quad-\frac{1}{\pi} \operatorname{Re}\left(\int_{0}^{\infty} \frac{\exp \left(\frac{2 \mu_{1}(a-x)}{\sigma_{1}^{2}}+\boldsymbol{i} u(a-x)\right)-\exp (-\boldsymbol{i} u(a-x))}{\boldsymbol{i} u} \phi_{T}(u) d u\right),
\end{aligned}
$$

where the characteristic function $\phi_{T}(u)$ is defined in Equation (2) and $\operatorname{Re}(z+i y)=z$ denotes the real part of the complex number $z+i y, z, y \in \mathbb{R}$.

(b) Consider two barriers $b<x=B_{0}<a$. If $\mu_{1}=0$, the double-barrier first-passage time is

$$
\mathbb{P}\left(T_{a b}^{+} \leq T\right)=\frac{x-b}{a-b}+K_{\Lambda_{T}}^{\infty}(b-x),
$$

for $\mu_{1} \neq 0$

$$
\mathbb{P}\left(T_{a b}^{+} \leq T\right)=\frac{\exp \left(-\frac{2 \mu_{1}(b-x)}{\sigma_{1}^{2}}\right)-1}{\exp \left(-\frac{2 \mu_{1}(b-x)}{\sigma_{1}^{2}}\right)-\exp \left(-\frac{2 \mu_{1}(a-x)}{\sigma_{1}^{2}}\right)}+\exp \left(\frac{\mu_{1}(a-x)}{\sigma_{1}^{2}}\right) K_{\Lambda_{T}}^{\infty}(b-x),
$$

where $K_{\Lambda_{T}}^{N}(k):=\frac{\sigma_{1}^{2} \pi}{(a-b)^{2}} \sum_{n=1}^{N} \frac{n(-1)^{n+1}}{\frac{\mu_{1}^{2}}{2 \sigma_{1}^{2}}+\frac{\sigma_{1}^{2} n^{2} \pi^{2}}{2(a-b)^{2}}} \vartheta_{T}\left(\frac{\mu_{1}^{2}}{2 \sigma_{1}^{2}}+\frac{\sigma_{1}^{2} n^{2} \pi^{2}}{2(a-b)^{2}}\right) \sin \left(\frac{n \pi k}{a-b}\right)$ and the Laplace transform $\vartheta_{T}(u)$ is given in Lemma 9.

\section{Proof}

The interested reader is referred to Hurd [2009], Hieber and Scherer [2012].

\section{Numerical comparison and applications}

The theoretical results are in this section compared to the Brownian bridge algorithm (originally by Metwally and Atiya [2002], extended to regime switching models by, e.g., Hieber and Scherer [2010]), to a finite elements scheme ${ }^{8}$ (see, e.g., Boyle and Draviam [2007],

\footnotetext{
${ }^{8} \mathrm{~A}$ possible implementation is given, e.g., in the Appendix of Kim et al. [2008].
} 
4 Numerical comparison and applications

\begin{tabular}{|c|c|c|c|c|}
\hline & \multicolumn{2}{|c|}{ Matrix Wiener-Hopf } & \multicolumn{2}{|c|}{ Brownian bridge } \\
\hline & $\mathbb{P}\left(T_{a,-\infty}<T\right)$ & time & $\mathbb{P}\left(T_{a,-\infty}<T\right)$ & time \\
\hline$L=1.4$ billion & $82.143 \%$ & $2 \mathrm{~ms}$ & $82.136 \% \pm 0.062 \%$ & $2.18 \mathrm{~s}$ \\
\hline$L=1.5$ billion & $61.999 \%$ & $2 \mathrm{~ms}$ & $61.952 \% \pm 0.079 \%$ & $2.19 \mathrm{~s}$ \\
\hline$L=1.8$ billion & $40.912 \%$ & $2 \mathrm{~ms}$ & $40.935 \% \pm 0.082 \%$ & $2.21 \mathrm{~s}$ \\
\hline \multirow[t]{3}{*}{$L=2.0$ billion } & $8.012 \%$ & $2 \mathrm{~ms}$ & $8.004 \% \pm 0.043 \%$ & $2.30 \mathrm{~s}$ \\
\hline & \multicolumn{2}{|c|}{ Finite elements } & \multicolumn{2}{|c|}{ Monte Carlo on grid } \\
\hline & $\mathbb{P}\left(T_{a,-\infty}<T\right)$ & time & $\mathbb{P}\left(T_{a,-\infty}<T\right)$ & time \\
\hline $\bar{L}=1.4$ billion & $82.088 \%$ & $5.18 \mathrm{~s}$ & $82.089 \% \pm 0.006 \%$ & $16.1 \mathrm{~min}$ \\
\hline$L=1.5$ billion & $62.126 \%$ & $5.17 \mathrm{~s}$ & $61.704 \% \pm 0.008 \%$ & $30.5 \mathrm{~min}$ \\
\hline$L=1.8$ billion & $40.844 \%$ & $5.15 \mathrm{~s}$ & $39.805 \% \pm 0.008 \%$ & $42.5 \mathrm{~min}$ \\
\hline$L=2.0$ billion & $7.849 \%$ & $5.18 \mathrm{~s}$ & $7.831 \% \pm 0.004 \%$ & $49.7 \mathrm{~min}$ \\
\hline
\end{tabular}

Table 1 The probability $\mathbb{P}\left(T_{a,-\infty}<T\right)$ that the Chinese population exceeds certain upper thresholds $L:=e^{a}$ by the year 2050 is given comparing the matrix Wiener-Hopf factorization (top left), the Brownian bridge algorithm (top right, $10^{7}$ simulation runs), a finite element scheme (bottom left, $\triangle t=10^{-3}, \triangle p=2 \cdot 10^{-2}$ ), and a brute-force Monte Carlo simulation (bottom right, $10^{7}$ simulation runs, mesh $10^{-3}$ years). The chosen parameters of the regime switching model are $1 / q_{11}=-26$, $1 / q_{22}=-100, \mu_{1}=1.484 \%, \mu_{2}=-0.341 \%, \sigma_{1}=\sigma_{2}=0.663 \%, T=2050-2010=$ $40, \mathbb{P}\left(Z_{0}=1\right)=1, a=\log (L), x=\log \left(P_{0}\right)$, and $P_{0}=1354815000$. The given computation time was calculated using Matlab on a $2.0 \mathrm{GHz} \mathrm{PC} ; 95 \%$ confidence intervals are given.

Kim et al. [2008]), and to a brute-force Monte-Carlo simulation on a grid. As an example, we consider the modeling of population growth (for similar studies using Brownian motion, see, for example, Lutz et al. [1997], Lutz et al. [2001]). Thereby we want to explicitly focus on structural break(s) that significantly and persistently change population growth.

Therefore, we assume that the vital rates follow a regime switching Brownian motion (model (1))

$$
d B_{t}=\mu_{Z_{t}} d t+\sigma_{Z_{t}} d W_{t}, \quad B_{0}=0
$$

where $Z_{t} \in\{1,2\}$. The evolution of the population is then described by $P_{t}=P_{0} \exp \left(B_{t}\right)$. We apply this model to real data on the Chinese population ${ }^{9}$. The initial year $t=0$ is 2010 with an estimated population of $P_{0}=1354815000$. We set $-1 / q_{11}=26$ (years) and

$$
\left(\begin{array}{l}
\mu_{1} \\
\mu_{2}
\end{array}\right)=\left(\begin{array}{r}
1.484 \% \\
-0.341 \%
\end{array}\right), \quad\left(\begin{array}{l}
\sigma_{1} \\
\sigma_{2}
\end{array}\right)=\left(\begin{array}{l}
0.663 \% \\
0.663 \%
\end{array}\right) .
$$

Table 1 gives some numerical results on the one-sided first-passage time probability in a 2-state model. The matrix Wiener-Hopf technique is compared to the Brownian bridge algorithm, to a finite elements scheme, and to a brute-force Monte-Carlo simulation discretizing

\footnotetext{
${ }^{9}$ The data is provided by the Statistics Division ESCAP of the United Nations and is publicly available on http://www.unescap.org/stat/data/.
} 


\section{Conclusion}

the underlying process $B$. For the matrix Wiener-Hopf approach, the Laplace transform in Theorem 5 was inverted ${ }^{10}$. This yields both the fastest (first-passage time probabilities are computed within fractions of seconds) and most accurate results. Although the simulation was performed on a very fine grid of 1000 data points per year, a significant discretization bias is observed for the brute-force Monte-Carlo simulation. The same problem arises for the finite elements scheme.

\section{Conclusion}

We showed how the Laplace transform of the regime-switching first-passage times can be derived in the 2- and 3-state model by solving the matrix Wiener-Hopf factorization analytically. If $\mu_{Z_{t}} / \sigma_{Z_{t}}^{2}$ is constant over the states, this Laplace transform can even be inverted, leading to rapidly converging infinite series. The matrix Wiener-Hopf factorization turned out to be both faster and more accurate than numerical alternatives (finite elements schemes, the Brownian bridge algorithm, or a brute-force Monte-Carlo simulation). Regime switching models are - unlike Lévy models - able to capture persistently changing trends in the underlying model parameters and have thus recently attracted considerable interest, for example, in macroeconomics, biology, physics, and finance.

\section{References}

J. Abate and W. Whitt. A unified framework for numerically inverting Laplace transforms. INFORMS Journal on Computing, Vol. 18, No. 4:pp. 408-421, 2006.

M. Abramowitz and I. A. Stegun. Handbook of Mathematical Functions. Dover Publications Inc., New York, 1965.

S. Asmussen. Stationary distributions for fluid flow models with or without Brownian noise. Communications in Statistics. Stochastic models, Vol. 11, No. 1:pp. 21-49, 1995.

F. Avram, M. Pistorius, and M. Usabel. The two barriers ruin problem vie a Wiener Hopf decomposition approach. Annals of University of Craiova, Vol. 30:pp. 38-44, 2003.

M. Barlow, L. Rogers, and D. Williams. Wiener-Hopf factorization for matrices. Lecture Notes in Mathematics, Vol. 784:pp. 324-331, 1990.

S. Boyarchenko and S. Levendorskii. Exit problems in regime-switching models. Journal of Mathematical Economics, Vol. 44:pp. 180-206, 2008.

10 There are many possibilities to numerically invert the Laplace transforms in Theorem 2; a good overview is given in Abate and Whitt [2006]. Note that some of those algorithms require high-precision arithmetic and can hardly be implemented using double-precision arithmetic. We could obtain reasonable results by numerically evaluating the integral

$$
\mathbb{P}\left(T_{a b}^{ \pm} \leq T\right)=\frac{\Psi_{a b}^{ \pm}(0)}{2}-\frac{1}{\pi} \int_{0}^{\infty} e^{-i u T} \frac{\Psi_{a b}^{ \pm}(-\boldsymbol{i} u)}{\boldsymbol{i} u} d u .
$$

If $\Psi_{a b}^{ \pm}(\cdot)$ has to be evaluated for purely imaginary numbers, a short proof shows that the order relation in Theorem $4-\infty<\beta_{1, u}<\beta_{2, u}<0<\beta_{3, u}<\beta_{4, u}<\infty$ has to be replaced by $-\infty<\operatorname{Re}\left(\beta_{1, u}\right)<\operatorname{Re}\left(\beta_{2, u}\right)<$ $0<\operatorname{Re}\left(\beta_{3, u}\right)<\operatorname{Re}\left(\beta_{4, u}\right)<\infty$. 


\section{References}

P. Boyle and T. Draviam. Pricing exotic options under regime switching. Insurance: Mathematics and Economics, Vol. 40:pp. 267-282, 2007.

J. Buffington and R. J. Elliott. American options with regime switching. International Journal of Theoretical and Applied Finance, Vol. 5:pp. 497-514, 2002.

N. Cai. On first passage times of a hyper-exponential jump diffusion process. Operations Research Letters, Vol. 37:pp. 127-134, 2009.

D. Darling and A. Siegert. The first passage problem for a continuous Markov process. The Annals of Mathematical Statistics, Vol. 24, No. 4:pp. 624-639, 1953.

R. J. Elliott, L. Chan, and T. K. Siu. Option pricing and Esscher transform under regime switching. Annals of Finance, Vol. 1, No. 4:pp. 423-432, 2005.

J. Folks and R. Chhikara. The inverse Gaussian distribution and its statistical application - a review. Journal of the Royal Statistical Society. Series B, Vol. 40, No. 3:pp. 263-289, 1978.

S. Fourati. Explicit solutions of the exit problem for a class of Lévy processes; applications to the pricing of double-barrier options. Stochastic Processes and their Applications, Vol. 122, No. 3:pp. 1034-1067, 2012.

X. Guo. When the "Bull" meets the "Bear" - A first passage time problem for a hidden Markov process. Methodology and Computing in Applied Probability, Vol. 3, No. 2:pp. 135143, 2001a.

X. Guo. An explicit solution to an optimal stopping problem with regime switching. Journal of Applied Probability, Vol. 38:pp. 464-481, 2001b.

J. D. Hamilton. A new approach to the economic analysis of nonstationary time series and the business cycle. Econometrica, Vol. 57, No. 2:pp. 357-384, 1989.

P. N. Henriksen. Pricing barrier options by a regime switching model. Journal of Quantitative Finance, Vol. 11, No. 8:pp. 1221-1231, 2011.

P. Hieber. A correction note on: When the "Bull" meets the "Bear" - A first passage time problem for a hidden Markov process. Methodology and Computing in Applied Probability, in press, 2013.

P. Hieber and M. Scherer. Efficiently pricing barrier options in a Markov-switching framework. Journal of Computational and Applied Mathematics, Vol. 235:pp. 679-685, 2010.

P. Hieber and M. Scherer. A note on first-passage times of continuously time-changed Brownian motion. Statistics 83 Probability Letters, Vol. 82, No. 1:pp. 165-172, 2012.

T. Hurd. Credit risk modeling using time-changed Brownian motion. International Journal of Theoretical and Applied Finance, Vol. 12, No. 8:pp. 1213-1230, 2009.

Z. Jiang and M. Pistorius. On perpetual American put valuation and first-passage in a regime-switching model with jumps. Finance and Stochastics, Vol. 12, No. 3:pp. 331-355, 2008. 


\section{References}

A. Jobert and L. Rogers. Option pricing with Markov modulated dynamics. SIAM Journal on Control and Optimization, Vol. 44:pp. 2063-2078, 2006.

J. Kalbfleisch, J. Lawless, and W. Vollmer. Estimation in Markov models from aggregate data. Biometrics, Vol. 39, No. 4:pp. 907-919, 1983.

J. Kennedy and D. Williams. Probabilistic factorization of a quadratic matrix polynomial. Mathematical Proceedings of the Cambridge Philosophical Society, Vol. 107:pp. 591-600, 1990.

M. Kim, B.-G. Jang, and H.-S. Lee. A first-passage-time model under regime-switching market environment. Journal of Banking \& Finance, Vol. 32:pp. 2617-2627, 2008.

S. G. Kou and H. Wang. First passage times of a jump diffusion process. Advances in Applied Probability, Vol. 35, No. 2:pp. 504-531, 2003.

O. Kudryavtsev. Efficient pricing options under regime switching. Working paper, 2010.

O. Kudryavtsev and S. Levendorskii. Fast and accurate pricing of barrier options under Lévy processes. Finance and Stochastics, Vol. 13, No. 4:pp. 531-562, 2012.

R. London, H. McKean, L. Rogers, and D. Williams. A martingale approach to some WienerHopf problems. Lecture Notes in Mathematics, Vol. 920:pp. 68-90, 1982.

W. Lutz, W. Sanderson, and S. Scherbov. Doubling of world population unlikely. Nature, Vol. 387:pp. 803-805, 1997.

W. Lutz, W. Sanderson, and S. Scherbov. The end of world population growth. Nature, Vol. 412:pp. 543-545, 2001.

S. Metwally and A. Atiya. Using Brownian bridge for fast simulation of jump-diffusion processes and barrier options. Journal of Derivatives, Vol. 10:pp. 43-54, 2002.

A. Mijatović and M. Pistorius. Continuously monitored barrier options under Markov process. Mathematical Finance, Vol. 23, No. 1:pp. 1-38, 2013.

E. Mordecki. Optimal stopping for a diffusion with jumps. Finance and Stochastics, Vol. 3, No. 2:pp. 227-236, 1999.

F. Oberhettinger and L. Badii. Tables of Laplace transforms. Springer Verlag, 1973.

D. Ratkowsky, J. Olley, T. McMeekin, and A. Ball. Relationship between temperature and growth rate of bacterial cultures. Journal of Bacteriology, Vol. 149, No. 1:pp. 1-5, 1982.

L. Rogers. Fluid models in queueing theory and Wiener-Hopf factorization of Markov chains. Annals of Applied Probability, Vol. 4, No. 2:pp. 390-413, 1994.

L. Rogers. Evaluating first-passage probabilities for spectrally one-sided Lévy processes. Journal of Applied Probability, Vol. 37:pp. 1173-1180, 2000.

L. Rogers and Z. Shi. Computing the invariant law of a fluid model. Journal of Applied Probability, Vol. 31, No. 4:pp. 885-896, 1994. 